\title{
APETITOS SALVAJES DE UN HOMBRE CIVILIZADO: EL MENÚ DE ROBINSON CRUSOE
}

\author{
THE SAVAGE APPETITES OF A CIVILIZED MAN: \\ ROBINSON CRUSOE'S MENU
}

\author{
Alba Daniela López Gamboa \\ Universidad Nacional Autónoma de México \\ Av. Universidad 3000, Ciudad de México, México \\ albadaniela_lg@comunidad.unam.mx
}

\section{RESUMEN}

La alimentación de Robinson Crusoe en la isla ilustra varios procesos evolutivos, que impiden afirmar que haya sido un hombre civilizado y domesticador durante su estancia en ella. Asimismo, su alimentación representa el proceso civilizador de la sociedad según las actividades propuestas por Lévi-Strauss de recolección de frutos, ganadería, preparación de pan y alimentos complejos. El consumo de ciertos alimentos, como el pan, carne, licores y frutos preservados, y las actitudes religiosas y discriminadoras ante los rituales y elementos alimentarios (como el rechazo al canibalismo y sus ayunos oratorios), permiten ubicar la alimentación de Crusoe en posiciones cambiantes del espectro dicotómico de la civilidad y la barbarie. 
Palabras clave: barbarie, civilidad, canibalismo, pan, estudios alimentarios.

\begin{abstract}
Robinson Crusoe's diet while on the island reveals evolutionary processes which prevent him reaching the status of a fully civilized man and domesticator. His diet also represents humanity's civilizing process via activities proposed by Lévi-Strauss, such as fruit collection, animal husbandry, bread making and the preparation of elaborate dishes. These activities as well as his consumption of certain foods such as bread, meat, liquor and preserved fruits, and his religious and discriminatory attitudes toward alimentary rituals and elements, such as cannibalism and celebratory fasts, position Crusoe's diet ambiguously within the civility/barbarism dichotomy.
\end{abstract}

Keywords: Barbarism, Civility, Cannibalism, Bread, Food Studies.

Recibido: 05/06/2019

Aceptado: 06/09/2019

\title{
I. INTRODUCCIÓN
}

Robinson Crusoe (1719) de Daniel Defoe retrata la vida del personaje homónimo que naufraga en una isla deshabitada, donde vive por veintiocho años. A lo largo de los trescientos años de su publicación, la novela se ha estudiado desde un sinfín de perspectivas. Se ha analizado atendiendo a cuestiones de colonización e imperialismo, como lo hace por ejemplo Christopher Flynn quien afirma que "Crusoe is placed on the island not to be an admirable individual, but to be the vanguard of a commercial and imperialist enterprise" (13). Otros la han abordado desde la religiosidad. Tal es el caso de Didier Bertrand, quien argumenta que Robinson Crusoe puede ser leída como literatura espiritual y se enfoca en las representaciones de distintas vertientes del protestantismo y las religiones orientales (29). Ambos autores consideran a Crusoe (o a Inglaterra, de quien deviene epítome) como una figura dominante en los respectivos ámbitos que trabajan. Sin 
embargo, en estas y otras aproximaciones críticas los alimentos no son considerados como parte esencial de la relación que Crusoe tiene con la isla o la civilización.

La comida y el canibalismo, sin embargo, han sido mencionados en los estudios robinsonianos ya que presentan una relación de poder entre hombres civilizados y hombres bárbaros: los primeros rechazan a los bárbaros porque estos consideran al hombre mismo como alimento y lo deshumanizan y, por ello, los hombres civilizados les despojan de su calidad de seres humanos y los ubican en el ámbito de lo monstruoso, lo animal o lo salvaje. Pero las relaciones de poder que el canibalismo presenta no se consideran en la novela desde los estudios alimentarios, sino desde las diferencias raciales, como sucede con Roxann Wheeler quien en su ensayo complejiza las nociones de raza en la novela como pertenecientes a un espectro no binario dentro de lo blanco y lo negro. Por otra parte, Lucinda Cole sí toca las cuestiones alimentarias, aunque de manera tangencial, cuando analiza el papel de la marcada ausencia de las ratas en la novela. No obstante, el enfoque de su trabajo no se inserta de lleno en el campo de los estudios alimentarios.

Si bien, estudiosos como Cole han señalado la relación que tienen los alimentos con las ratas y la civilidad, es poco frecuente encontrar perspectivas críticas que aborden lo que en la novela se ostenta como la superioridad de lo civilizado ante la naturaleza barbárica desde el punto de vista de los estudios alimentarios. Así pues, en las siguientes páginas, analizaré las ya revisadas relaciones de civilización y barbarie, desde ese punto de vista. Mi propuesta es que la comida presentada y consumida por Crusoe en la isla refleja la posición ambigua que ocupa en ese esquema dicotómico. Los productos destinados a la ingesta constituyen el eje principal desde el que se analizará la barbarie de la isla (representada en las frutas, raíces y las cabras salvajes); el intento de Crusoe de domesticar y civilizar el territorio (por medio de la caza con armas y el progreso de las técnicas de cocción de alimentos); y al final, su reafirmación como ser civilizado al repudiar las prácticas caníbales. Este trabajo no busca categorizar a Crusoe como un hombre barbárico, sino como uno cuya postura dinámica, marcada por sus 
hábitos alimenticios, oscila por momentos entre la civilidad y la barbarie. Todo esto tiene la finalidad de complementar y matizar las discusiones sobre la civilidad intrínseca de Crusoe.

\section{Alimentos europeos}

Los primeros alimentos que Crusoe come al llegar son ajenos a la isla. El náufrago hace énfasis en la sensación de desamparo que le produce el distanciamiento de las comodidades conocidas: "I had a dreadful Deliverance: For I was wet, had no Clothes to shift me, nor any thing either to eat or drink to comfort me, neither did I see any Prospect before me, but that of perishing with Hunger, or being devour'd by wild Beasts" (Defoe 35-6). Más tarde encuentra agua fresca, que complementa con tabaco para evitar sentir hambre (36). Así pues, no come nada hasta que logra subir al barco, donde encuentra que "all Ship's Provisions were dry and untouch'd by the Water, and being very well dispos'd to eat, I went to the Bread-room and fill'd my Pockets with Bisket, and eat it as I went about other things" (37). Es importante notar que antes de rescatar objetos y establecer su morada en la isla, lo primero que hace es comer pan europeo. Esto parecería sugerir que la isla en su estado puro es inapropiada para alimentar a Crusoe, o, incluso que él se aferra a consumir solo lo familiar (y europeo), incluso en caso de necesidad.

Después de comer bisket y ron en su primer día, Crusoe rescata otros alimentos, con los que se nutre durante los primeros meses. Estos representan un intento por civilizar su dieta incluso antes de que pueda barbarizarla. Por ser europeos, no solo sacian el hambre inicial de Crusoe, sino que lo reconfortan al relacionarlo con su sociedad natal, y le ayudan a recuperarse del impacto inicial del naufragio. De este modo, Crusoe mantiene su cotidianidad alimentaria; es decir, una dieta marinera que se basa principalmente en la carne seca y las galletas.

Estos alimentos, a pesar de no ser consumidos de manera habitual por la población inglesa, se derivan de elementos europeos que, por 
extensión, se considerarían como civilizados (pan, queso y carne). Cabe notar, sin embargo, que, desde la vida marinera, se puede observar una degradación ostensible respecto de las prácticas inglesas, puesto que los alimentos no son frescos. No obstante, Crusoe parece pasar por alto esta sutil ironía.

Me referiré, entonces, a los alimentos europeos no como civilizados, sino como tendientes a lo civilizado. El progreso de la narración sugiere que los alimentos clasificados de este modo cumplen con ciertos estándares de obtención (recuperación), producción, cocción y preservación. En esta categoría están aquellos rescatados del barco: queso, galletas náuticas, pan y licor. También entran en ella los producidos más adelante en la isla, como el pan de cebada -producto del cultivo y manufactura del grano en la isla-, las pasas - preservadas por Crusoe- y los productos derivados de las cabras una vez que han sido domesticadas (carne y leche).

La comida inicial de Crusoe deja ver su impulso europeizante del espacio, puesto que es elaborada a base de "Bread, Rice, three Dutch Cheeses, five Pieces of dry'd Goat's Flesh [...] European Corn [...] some Cordial Waters, and in all about five or six Gallons of Rack" (38). Dado que las primeras provisiones que Defoe decide poner al alcance de su protagonista son alimentos comunes de la sociedad europea, la novela parecería sugerir que la mejor manera de saciar el impulso más básico de hambre es por medio de productos que guardan una fuerte relación con lo que, desde el ángulo europeo, constituye lo civilizado, como es el pan.

\section{Pan europeo y pan isleño}

El pan era considerado indispensable en cada comida europea dieciochesca. En sí misma, la palabra pan evoca vida en sociedad, pues el significado etimológico latino de compañero y compañía es compartir pan (Albala 21). Durante gran parte de su estancia, Crusoe consume pan, al inicio el que rescata y después el que fabrica. En etapas posteriores del relato, cuando salva prisioneros en los últimos años de su vida en la isla lo primero que 
les ofrece es pan, ron y agua, como señal de compañerismo. Esta conducta deviene en una marca de pertenencia, una forma de mostrar tanto a otros personajes de la historia, como a los lectores que, a pesar de haber estado en soledad y en un ambiente salvaje durante veinticuatro años, continúa poseyendo el alimento epítome de la convivencia humana. Y es que, para las culturas indoeuropeas, el pan es la marca de una sociedad civilizada. De acuerdo con Massimo Montanari:

[Bread] symbolizes man's exit from the animal kingdom and the establishment of "civilization." In the epic poems The Illiad and The Odyssey, "bread eaters" are synonymous with "men." Similarly, the epic of Gilgamesh, the first literary text known [...] tells of a "wild man" who left his status as a minor only at the moment when he learned of the existence of bread. (6-7)

Entonces, el pan separa al protagonista de manera automática del ámbito animal. Pero una vez agotado el pan europeo, el náufrago se ve obligado a elaborar pan con cebada, usando técnicas rudimentarias, lo que barbariza su producto, aunque el protagonista nunca lo admita de este modo. Leer atentamente esta relación de Crusoe con el pan es, entonces, un elemento clave que problematiza su posición en la novela, pues se observan los matices y el dinamismo con el que se mueve entre el ser civilizado o barbárico respecto de su alimentación.

El tipo de pan que Crusoe consume durante sus primeros años en la isla son las galletas náuticas. Esta bollería es un reflejo de su alimentación en la otra isla, en este caso civilizada, de donde proviene: Inglaterra. Como lo explica Albala, dado que la dieta europea se basa en el consumo de trigo y otros granos, "dried biscuits or hard tack bread [were] carried on long ocean voyages" (67), su consumo en el barco implica adaptar la dieta civilizada en un ambiente ajeno a la sociedad de origen, y Crusoe extiende esta adaptación al llevar estos alimentos -ya adaptados- a la isla, con lo que, desde ese punto de vista, conseguiría civilizar su dieta de forma parcial. Las galletas náuticas, en inglés llamadas biscuits (o bisket, como se le denomina en la novela) son alimentos cocidos. De hecho, si consideramos 
su etimología, lo son doblemente. Es importante notar que las galletas náuticas son transportadas en el barco y comidas ahí mismo, ya cocidas. Estos alimentos europeos cocidos separan a Crusoe del ámbito barbárico (que sería, entre otros, comer alimentos crudos), pero como Crusoe no las cuece y depende de este sustento limitado, también ilustran la precariedad de su estado de civilización. Parte de su dieta coincide temporalmente con aquella que hubiera tenido en su sociedad natal. El pan sobrevive a la tormenta y a las ratas, pero aun si la provisión ha sido casual, Crusoe decide rescatar este alimento en particular como se ve en su listado "Bread, Rice, three Dutch Cheeses" (38). Sin embargo, Crusoe no produce el mismo tipo de pan, ni las galletas náuticas que consume al inicio de su estancia, por lo que esta actividad alimentaria no es total ni estable: su alimentación depende de un sinfín de factores que a su vez involucran una cierta pertenencia a lo civilizado o lo barbárico, dependiendo del alimento y de lo que lo rodea. Por ejemplo, en cuanto al método de preparación de las galletas, según Lévi-Strauss "cooking marks the transformation from nature to culture" (The Raw 164). Y en este sentido, aun si las galletas representan lo civilizado (culture) y separan a Crusoe de lo salvaje (nature), desde los primeros momentos en que gozaría de un supuesto bienestar al consumir provisiones europeas, se mueve en un área ambigua, pues come algo que él no hizo fuera del lugar en que fueron cocinadas.

\section{Crusoe agricultor}

Si el pan es el alimento epítome de la civilización, es relevante trazar la evolución de su ingrediente principal: los granos. Es importante puntualizar que durante el tercer y cuarto año de su estancia, Crusoe no consume pan, pues las galletas náuticas se le agotan antes de que produzca el suyo propio. Su falta de contacto con lo civilizado lleva a Crusoe a realizar su primer acto importante como agente civilizador, esto es, cultivar granos. Los granos en su estado no procesado son los únicos alimentos que transmiten un sentido de continuidad, puesto que logra comerlos de forma ilimitada 
desde que los rescata del barco, más tarde cuando los cosecha y hasta el final de su estancia.

El consumo de pan, sin embargo, no presenta la misma estabilidad. Cuando se le agotan las galletas, Crusoe tiene que esperar a cultivar los granos rescatados antes de hacer pan. Dado que el pan es uno de los parámetros fundamentales para considerar a una sociedad como civilizada (Montanari 6-7), la carencia temporal de este podría considerarse como un momento de retroceso, o pausa, en la evolución alimentaria de Crusoe en la isla, hacia lo barbárico.

Meses después del naufragio Crusoe descubre que de la tierra han brotado "ten or twelve Ears [...] [of] perfect Green Barley of the same Kind as our European nay, as our English Barley" (Defoe 58), que, a sus ojos, constituyen una suerte de milagro y lo llevan a pensar que "God had miraculously caus'd this Grain to grow without any Help of Seed sown, and that it was directed purely for my Sustenance" (58). Este evento -que el protagonista califica de milagroso- funciona como justificación del derecho que cree tener de civilizar la isla y sus elementos silvestres. Como afirma LeBlanc: "Robinson Crusoe constructs $[\ldots]$ the 'other' as [a] savage cannibal in order to have him[self] serve as the direct hero, who is a civilized European man of culture" (75), lo cual provoca que "Crusoe's narrative account of his adventures is told from the culturally, and sexually biased perspective of an empowered White Anglo-Saxon male from imperialist Europe" (75). El protagonista opera como imagen de civilización, la que se anuda con la idea del pan que ingiere como alimento civilizador.

Este milagro constituye un parteaguas para la narrativa de domesticación y europeización, puesto que la vida de Crusoe y su dieta se ven transformadas por completo. La aparición de las espigas de cebada y arroz permite tres cosas: la variación en sus comidas, la producción de pan y, por extensión, un mayor grado de civilización alimentaria. La variación en su dieta se logra ańos después de descubrir las espigas, cuando al fin logra conquistar la naturaleza. Una vez que obtiene el cereal, se lo agrega a sus caldos, espesándolos y convirtiéndolos en estofados, también los muele y cuece para 
hacer pan (90). Así, al incluir estos dos platillos en su dieta, logra acercarse a aquella que hubiera tenido en Inglaterra, lo que le permite civilizar la isla.

Es importante recalcar el porqué Defoe se toma la molestia de retratar este arduo proceso panadero. La mención de los procesos y lo lento que resulta concretarlos ilustra cómo la superioridad tecnológica de un hombre europeo, aunada a su frugalidad y perseverancia, son capaces de domar a la naturaleza salvaje. Por otro lado, la ironía de que el papel civilizatorio y dominante sobre la naturaleza barbárica no es concretado por Crusoe hasta el final de la novela y que este proceso, a pesar de responder a la aspiración de realismo en la novela dieciochesca, no puede ser fácil ni perfecto.

La incipiente agricultura de estas espigas podría considerarse reflejo de la primera etapa de la civilización. Según Montanari, "agriculture [is] the moment of breakthrough and innovation [...] the decisive leap from "civilized" man, separating him from nature" (4). La evolución que presenta Crusoe es correlato de ello, pues primero cultiva y luego domestica animales. Del mismo modo, esta narrativa de progresión simboliza su papel -autoasumido- de civilizador, al iniciar la revolución de la isla, tras conquistarla con la introducción de una agricultura primitiva. El cultivo de cebada y el arroz es, entonces, el primer paso que Crusoe logra dar en la domesticación de la isla.

\section{LA AMBIgÜEdAd DE LA UVA PASA}

Otra domesticación de alimentos que Crusoe implementa es la preservación de las uvas en forma de pasas. Este producto, que el náufrago obtiene antes de poder manufacturar su pan isleño, denota su incipiente civilización alimentaria. El descubrimiento de las uvas y la descripción del secado se hace unos meses después de su llegada a la isla:

I found [...] Grapes upon the Trees [...] [And] I found an excellent Use for these Grapes, and that was to cure or dry them in the Sun, and keep them as dry'd Grapes or Raisins are kept, which I thought would be, as indeed they were, as wholesom, as agreeable to eat, when no Grapes might be to be had. (73) 
Esta es la primera mención de una técnica de preservación de alimentos. La forma más común de preservar era el secado, que se hacía al sol (Montanari 16), técnica que Crusoe replica con las uvas para dosificar su consumo a lo largo del ańo. Crusoe tiende así hacia lo civilizado, puesto que va más allá de la producción básica de comida y permite conservar los frutos después de su temporada. Por otra parte, uno de los elementos rescatados del barco es la carne seca de cabra. Crusoe, entonces, continúa el patrón de recrear su dieta inglesa en la isla, pues al secar la uva en forma de pasa, recurre a la misma técnica de preservación que la usada en el barco.

La uva es salvaje, crece sin orden y sin la intervención de Crusoe. Su presencia en la isla es por completo indómita, por lo que el personaje, a pesar de beneficiarse del consumo de esta fruta, se ve perjudicado implícitamente en su estatus autoasumido de no salvaje, al consumir alimentos silvestres que crecen sin control y adquirir un salvajismo parcial. Aun si los europeos consumen frutas naturales (y la uva es una de ellas), las uvas de la isla de Crusoe no se cultivan y, por lo tanto, no responden a un esfuerzo civilizador.

La presencia de la uva en la novela puede leerse desde, al menos, dos perspectivas: la edénica y la salvaje. La fruta que crece sin apoyo y para beneficiar únicamente al hombre, se presenta, como en el caso del jardín del Edén, como derecho consumible. La isla, en este aspecto, representaría una suerte de Edén, al proveer a Crusoe de sustento disponible y abundante, pero también es importante considerar que consumir frutos salvajes no era algo común en la sociedad que Crusoe intenta replicar.

Hay que recordar que las tradiciones y costumbres alimentarias inglesas no son estáticas, sino que van cambiando de siglo en siglo, y el XVIII no fue la excepción. Por ejemplo, Newman describe la situación dietética de los siglos XVI y XVII y advierte que "[b]arbaric and elaborate meals including many strange dishes with strong and almost incredible flavouring gave way to a more natural selection of foods, richer in quality, but otherwise little different from the diet and cookery of the present time" (39). Newman se refiere a los alimentos de ese período como barbáricos con leves modificaciones. Esto apunta a que, aun si se cambiaron ciertos aspectos de ellos, en esencia continúan siendo alimentos que, desde un 
punto de vista occidental evolucionista y tecnológico, son barbáricos. Esta noción del alimento barbárico cambia en el siglo XVIII.

El alimento barbárico es esencialmente un elemento transgresor, ya sea de acuerdo con estándares de raza, civilización, o de posición social. Montanari y Albala mencionan que la dieta de los pobres dependía en gran medida de animales y plantas locales y salvajes (Montanari 19, Albala 6). Crusoe, al consumir alimentos salvajes en la isla, transgrede su estrato social de clase media inglesa y su autoimpuesto título de rey de la isla. Su dieta en este aspecto es barbárica en comparación con la que debería de llevar en ambos casos.

Así, la dicotomía civilidad/barbarie alimentaria está presente tanto en la ingesta de la fruta salvaje al descubrirla, como en su decisión de preservarla y, en cuanto le es posible, comerla exclusivamente en su estado conservado. A pesar de disponer de uvas frescas, una vez que logra secarlas, prioriza el consumo de las pasas. Entonces la uva, a pesar de haber sido recolectada (y comida al momento de su descubrimiento) como fruto salvaje, ha sido domesticada por medio de su preservación, y es comida después de ser procesada, desplazando el alimento de su origen salvaje (por su obtención) hacia un consumo civilizado (por su preservación). En su desarrollo civilizador de la isla, Crusoe respeta la progresión observada por teóricos como Albala, quien apunta que "the first major transformation ocurred when our ancestors gradually left their hunting and gathering lifestyle for settled agriculture and cattle raising" (6). Así, tras iniciar su proceso civilizador con la agricultura, sin abandonarla, progresa hacia la ganadería.

\section{Crusoe ganadero}

El pan europeo que Crusoe consume al inicio de su estancia y el licor que bebe durante ella, aunque importantes en su dieta, no son el sustento principal de sus comidas, como sí lo es la carne, pues "meat was the preferred type of animal protein among all classes and second only to bread in terms of its centrality to the British diet" (Durbarch 966). Aquí comienza el 
barbarismo alimentario, puesto que no puede recuperar carne fresca entre sus provisiones y debe recurrir a la caza.

En culturas que no son nómadas, la caza con armas de fuego es una actividad lúdica, asociada a las clases dominantes, y considerada como civilizada. Sin embargo, dado que para Crusoe es una necesidad, se convierte en una actividad cuasibarbárica. El grado de civilización de la caza-que a ojos de los contemporáneos de Crusoe implica el avance tecnológico de las armas usadas y la actividad misma- está asociado a situaciones de esparcimiento y no de supervivencia. Esta diferencia circunstancial permite contrastar sociedades en los modos de obtener los alimentos, puesto que

in the historical developments of human societies, a 'domestic' economy based on agriculture and livestock raising contrasts with a primitive, undomesticated economy in the acquisition of food: raising animals, or hunting them; cultivating the fruits of the earth, or picking them in their wild state. (Montanari 9)

Si se aplica esta visión en la isla, la evolución civilizadora que Defoe aspira a mostrar en la novela puede trazarse desde el momento en que el protagonista recolecta las uvas silvestres hasta su transformación en una sociedad doméstica y ganadera.

Su proceso ganadero, sin embargo, presenta una leve regresión. A pesar de que la ganadería es un avance hacia la civilidad alimentaria, en cuanto al proceso evolutivo de obtención de alimentos, Crusoe la aborda de manera inversa. La provisión de carne inicia primitivamente al ser cazada y no criada. Antes de la cría de animales no se puede considerar que comer carne es un avance civilizador en su dieta, pues el comer animales salvajes se relaciona con el salvajismo adquirido, al igual que consumir las uvas frescas. Así, Crusoe transgrede los estándares sobre el consumo de animales que su sociedad hubiera considerado como civilizados al cazar cabras salvajes y comer su carne. A pesar de que "kid was considered among the healthiest meats of all by physicians, especially those who depended on medieval Arabic authorities like Avicena and Averröes" (Albala 47), 
este alimento no mantiene su estándar preferencial. Montanari y Albala respetan la autoridad medieval y, por lo mismo, sus argumentos remarcan la ambigüedad cárnica de Crusoe. La cabra, a pesar de ser considerada saludable, no representa una civilidad alimentaria, puesto que su origen silvestre permea a quien la consume.

Así, el último elemento alimentario que logra concretar Crusoe es la ganadería caprina. El protagonista de la novela progresa hacia el siguiente paso de la evolución propuesta por Albala. Entonces, su alimentación puede ser matizada según los contrastes que presenta. Lévi-Strauss considera que

a difference in cultural level is observed between this Society and those to which it may be most easily compared. Its culture is poorer, owing to the absence or inadequacy of those features - permanent dwellings, horticulture, animal husbandry, techniques of stone-polishing, weaving and pottery-. (Structural Anthropology 122)

Las sociedades que comparo son la de Inglaterra dieciochesca y la de la isla de Crusoe. La horticultura es rudimentaria y susceptible; no logra domesticar animales sino hasta once años después de llegar a la isla, ya que cuando Crusoe resuelve que "being now in the eleventh Year of my Residence [...] I set myself to study some Art to trap and snare the Goats" (105), presenta una conciencia ganadera que supera su primer estado de agricultor.

Aunque la carne es el único alimento que Crusoe procesa antes de comerlo, la cocción civilizadora no es suficiente para contrarrestar su origen silvestre. El consumo de carne había sido parcialmente salvaje -en tanto que las armas que usaba para cazar y la cocción del alimento antes de su ingesta la alejaban en cierta medida de lo barbárico- y solo a partir del decimoprimer año de su estancia - momento en que comienza a comer las cabras que ha domesticado- tiende hacia un estado civilizado. Este movimiento se refleja en Crusoe mismo, quien, al abandonar elementos totalmente salvajes en su dieta, puede alejarse de ese extremo de la dicotomía. Asimismo, esta evolución alimentaria junto con la domesticación de los elementos dietarios 
imita la secuencia de desarrollo de la civilización humana señalada por Montanari: primero la agricultura y luego la ganadería.

\section{Crusoe cocinero}

El consumo de carne en la isla también representa una evolución que está relacionada con las técnicas de cocción. Al inicio Crusoe solo puede asar la carne, con el tiempo la hierve y después, gracias a la cosecha de granos y perfección de sus técnicas culinarias, logra estofarla. Este proceso culinario se nota en las menciones de cocina que hace Crusoe en su diario: "June 26 [...] I kill'd a She-Goat [...] broil'd some of it, and eat; I wou'd fain have stew'd it, and made some Broath, but had no Pot" (64); luego con "I brought home the Kid [...], and having a Pot for that purpose, I boil'd or stew'd some of the Flesh, and made some very good Broth" (153); hasta "I order'd Friday to take a yearling Goat [...] and chopping it in small Pieces I set Friday to Work to boiling and stewing, and made them a very good Dish, I assure you, of Flesh and Broth, having put some Barley and Rice also into the Broth" (174). La transición en las preparaciones establece un paralelismo con su grado de civilización alimentaria en los momentos descritos y refleja un cambio en los métodos por medio de los artefactos para cocinar.

Por falta de cazuelas, a su llegada a la isla, Crusoe se ve forzado a asar su carne. Esto marca el inicio de su evolución culinaria, pues no tiene utensilios para sofisticar su cocción. El asar alimentos liga a Crusoe a un lugar de barbarismo culinario, pues "roasting decisively remains an expression of 'nature' and the 'wild,' since the only means of cooking is a fire over which the meat is cooked directly" (Montanari 47-8). Su método de cocción es primitivo, lo que primitiviza su consumo de carne. Sin embargo, es importante reconocer que el platillo inglés por excelencia es el asado, o roast beef, un guisado que denota su cultura. Durbach se refiere a "beef-and high-status blood-rich roasted beef in particular-was almost universally acknowledge to be the quintessential food of the English people" (968). 
Entonces, es importante cuestionar si Crusoe está replicando el modo de vida británico en sus decisiones culinarias o si este paso en su evolución responde simplemente a una necesidad de comer alimentos cocidos en concordancia con las restricciones materiales.

El asado inglés, a pesar de ser natural en esencia, como apunta Montanari, es civilizado en la medida que se pueda elegir cocer la carne de otras maneras más sofisticadas. Crusoe vuelve a caer en una contradicción en su estado implícito como epítome de lo inglés al sustituir el asado en cuanto le es posible. Una vez que logra fabricar cazuelas, hierve la carne, la cual

"mediates" through water the relationship of fire and food and necessitates the use of a container-utensil -namely, a manufactured object that typically represents 'culture' -thus tending to take on symbolic meanings more directly linked to the notion of "domestication". (Montanari 48)

Crusoe elige un método de cocción por encima del otro, modificando sus platillos. Irónicamente, mientras avanza en una línea de complejidad técnica, se aleja de la dieta inglesa. Así, al tener tanto cazuelas y granos, logra estofar su carne. Estofar está "only one step away from boiling [...] boiled foods are served separate from the cooking liquid [...] stewed foods are left in the cooking medium which is usually reduced and thickened" (Albala 63). Estofar, al ser el último método de cocción, es, entonces, la cúspide de la técnica culinaria de Crusoe. Su refinamiento en la cocina refleja su alejamiento gradual del barbarismo alimentario: primero, deja de consumir uvas sin preservar; segundo, cultiva granos; tercero, domestica cabras; y, por último, refina sus técnicas culinarias.

\section{ESPOSO DEL LICOR}

El último elemento de su dieta, y el que más dura a lo largo de su estancia en la isla, es el licor. Las bebidas alcohólicas figuran al final de su lista de alimentos rescatados del barco en su naufragio: "For liquors, I found 
[...] some Cordial Waters, and in all about five or six Gallons of Rack" (38). El ron era "one of the most important and valuable commodities in eighteenth-century Atlantic trade” (Albala 59) lo que explica el valor que Crusoe le da: es terapéutico en tanto que lo usa, junto con el tabaco, como remedio para su fiebre (69) y como tónico calmante para Friday ("I went and fetch'd a good Dram of Rum, and gave him [Friday]; for I had been so good a Husband of my Rum, that I had a great deal left" (167)). Crusoe se refiere a sí mismo como esposo del licor y le da un papel preponderante en su vida cotidiana. Es relevante mencionar que la palabra husbandry en el siglo XVIII se usaba para denotar la actividad agricultora o granjera (The Complete Farmer). Sin embargo, también podríamos leer el uso de este término como sustituto simbólico de las relaciones romántico-afectivas ausentes en el relato. Crusoe cuida y se hace acompañar por el licor: lo lleva consigo a las exploraciones de la isla, no lo deja atrás al mudarse de vivienda, lo carga en sus batallas contra los caníbales y lo da como ofrenda de amistad a quienes rescata y hace sus súbditos.

Si bien este apego al licor podría sonar exagerado, el nivel de consumo de Crusoe no corresponde a la idea del salvaje alcohólico que tenían los europeos de las sociedades coloniales (y barbáricas) en el desenfreno ante el consumo de sustancias. Joan Fitzpatrick aborda el concepto en la figura de Calibán, quien es "naïve enough to believe that Stephano would make a Good master and [his] wanton appetite is evident in his attempted rape of Miranda and possibly also in his eagerness to drink the wine offered to him by Stephano" (132). Así, el amerindio se describe como alguien incapaz de controlar sus apetitos sexuales, alcohólicos y alimentarios. A su vez, se creía que un hombre civilizado europeo, al momento de habitar en una colonia, estaba en riesgo de contagiarse de esta incapacidad. Crusoe no se excede y tampoco lo hace Friday, que contrasta con Calibán, el otro salvaje literario, representante de la ebriedad descontrolada.

Por su parte, la cerveza tiene relevancia en la novela. Crusoe hace hincapié en sus deseos de consumir cerveza y lamenta su imposibilidad de producirla: 
I had dropp'd a good Design, which I had once bent my Thoughts too much upon; and that was, to try if I could not make some of my Barley into Malt, and then try to brew my self some Beer: This was really a whimsical Thought, and I reprov'd my self often for the Simplicity of it; for I presently saw there would be the want of several Things necessary to the making my Beer, that it would be impossible for me to supply; as First, Casks to preserve it in, which was a Thing, that as I have observ'd already I cou'd never compass; no, though I spent not many Days, but Weeks, nay, Months, in attempting it, but to no purpose. In the next Place, I had no Hops to make it keep, no Yeast to make it work, no Copper or Kettle to make it boil. (121-2)

Esta carencia marca su ambigüedad alimentaria, pues no puede concretar una dieta totalmente civilizada, porque no consume la bebida básica de la sociedad europea del siglo dieciocho en que la cerveza "[was] not only a drink for social occasions but a regular food” (Albala 58). Consumirla acercaría al personaje a la dieta común de su sociedad natal y al no hacerlo queda al margen de la civilización, a la que, como hemos visto, se ha ido acercando de forma gradual. Este impedimento y la pausa que implica en su evolución alimentaria imposibilita que Crusoe se posicione por completo en el extremo civilizado de la dicotomía alimentaria. Como ha comido -hasta ese momento-productos lo suficientemente civilizados, no pierde su estado de civilidad parcial, habiendo superado su barbarie previa, pero se estanca momentáneamente al no avanzar más en cuanto a la comida y no acceder a bebidas civilizadas.

De ser producida y consumida en la isla, la cerveza funcionaría como el ejemplo principal del grado último de la civilización de Crusoe, dado que su preparación involucra la cosecha de cebada, que ilustra la práctica de la agricultura, y posteriormente las técnicas de molienda de grano y la fermentación. Como las bebidas fermentadas "represent an outgrowth of knowledge and of complex technology” (Montanari 7), la cerveza sería el epítome civilizado de las técnicas que Crusoe ya ejecuta, pero por falta de elementos muy puntuales, como el cobre o el lúpulo, no puede elaborarla. 
Si bien tomar cerveza no es en sí mismo reflejo de mesura, Crusoe demuestra a lo largo de la novela una conducta moderada hacia la comida, lo que anuda esta actitud a todas las acciones alimentarias, incluyendo la ingesta de bebidas alcohólicas. Entonces, el anhelo de cerveza y su probable mesura a la hora de beberla reflejan el antojo de un hombre civilizado y británico. No producirla impide que logre concretar este deseo civilizatorio, manteniéndolo en una posición parcial en esa dicotomía.

\section{Crusoe protestante}

Las creencias religiosas de Crusoe permean sus aproximaciones a la comida. Por ejemplo, en sus aniversarios de naufragio, en vez de tener un festín, se abstiene de la comida. Pasa su día en ayuno y oración:

I was now come to the unhappy Anniversary of my Landing. I cast up the Notches on my Post, and found I had been on Shore three hundred and sixty five Days. I kept this Day as a Solemn Fast, setting it apart to Religious Exercise $[\ldots]$ and having not tasted the least Refreshment for twelve Hours, even till the going down of the Sun, I then eat a Bisket Cake, and a Bunch of Grapes, and went to Bed, finishing the Day as I began it. (Dafoe 76)

La observación religiosa se presenta aquí en forma de ayuno. Para los cristianos, el ayuno es el sacrificio del cuerpo para acercarse a la experiencia de Cristo, o la preparación del cuerpo para recibir a Cristo (Grumett 161; McLean 268). La abstinencia de Crusoe refleja no solo su superación de la necesidad alimentaria, al renunciar voluntariamente a la comida, sino también un estado de profunda devoción. Crusoe ayuna en el aniversario de su naufragio, ora con frecuencia y estudia la biblia todos los días (Defoe 84). Su estancia en la isla gira en torno a rutinas, las que no solo incluyen actividades necesarias para su sustento, sino también actividades que reflejan su devoción cristiana. 


\section{IO. EUCARISTÍA ROBINSONIANA}

En cuestión de alimentos, el pan funciona como conector entre la observación religiosa de Crusoe, su civilidad y su temor ante los caníbales. Este alimento puede llegar a tener una fuerte carga religiosa en tanto que se relaciona con uno de los rituales cristianos que más controversia suscita entre las diferentes denominaciones: la eucaristía. Para los católicos, cuyo dogma afirma la transubstanciación (es decir la transformación del cuerpo de Cristo en pan y su sangre en vino), la eucaristía es la parte principal de la misa. Un ritual similar, pero que toma la transubstanciación de forma simbólica y no literal, se presenta también en ciertas ceremonias protestantes.

A pesar de sus diferencias, la eucaristía es un momento de ingesta. Como lo expuso Sophie Gee ${ }^{1}$, la eucaristía protestante que Crusoe practica supera tanto al ritual canibalístico de comer carne como al canibalismo virtual de la transubstanciación católica, para en su lugar asegurar el valor del sacramento protestante, el que posibilita la comunicación entre seres humanos a través del acto social de consumir pan y vino. La invalidación de la transubstanciación eucarística del protestantismo involucra repudiar el canibalismo real y el virtual. El desprecio que Crusoe exhibe hacia lo que él denomina, de manera despectiva, papismo permea su rechazo absoluto ante el canibalismo, pues se niega a representar el consumo de carne humana simbólicamente.

\section{i i. Canibalismo}

Defoe se esfuerza por hacer notar a sus lectores que la forma y el tipo de ingesta de comida de los salvajes o los otros, los acerca hacia lo animal. Desde luego Crusoe, dado que se ofrece a sí mismo como representante de lo civilizado, a lo largo del relato hace patente su distanciamiento de

En el marco del congreso "De náufragos emprendedores y pasiones desbordadas" realizado en mayo de 2019 en la Universidad Nacional Autónoma de México. 
los salvajes y de los animales, como se ejemplifica en su encuentro con el festín caníbal. Crusoe no lo presencia de manera directa, sino que encuentra las sobras que evidencian su culminación. Ante esto, se niega a comer la carne humana que allí encuentra (a pesar de que en ese punto del relato había tomado y transformado todo tipo de alimentos ofrecidos por la isla), con lo que se separa de las bestias come-humanos que son los caníbales, y también desdeña la idea de consumir sobras de comida, como lo hacen los animales.

Hasta ese punto de la novela, Defoe retrata la aparición de la comida de forma tal que simula una serie de encuentros oportunos con alimentos que están casi listos para ser consumidos, por lo que es relevante notar que los restos de cuerpos son presentados de la misma forma. Aun si las descripciones son grotescas ("The Blood, the Bones, and part of the Flesh of humane Bodies, eaten and devour'd by those Wretches, with Merriment and Sport", 133), se describen de la misma manera que los otros alimentos que Crusoe encuentra.

El repudio de Crusoe a las ceremonias pseudocaníbales del catolicismo, se extiende hacia el canibalismo ceremonial de los salvajes. Crusoe está obsesionado con el peligro de ser comido. Su actitud hacia los caribeños, que él considera como el pueblo salvaje, es analizada por Cătălin Avramescu, quien afirma que

In Defoe, we find a complete discussion of the right to declare war on the cannibals. Discovering the remains of the cannibal feast on his island, Robinson can think only of nothing but how "he might destroy some of these Monsters". (118-9)

Avramescu describe el momento en que Crusoe encuentra los cuerpos desmembrados en la playa (133), ante lo cual él "was so fill'd with Indignation at the Sight, that I began now to premeditate the Destruction of the next that I saw there, let them be who, or how many soever" (133). Este es el momento cúspide de su civilidad alimentaria. Tanto su reacción visceral, su intención de venganza y su abstinencia del alimento pertenecen 
al ámbito de lo que el personaje y la sociedad inglesa dieciochesca juzgan como civilizado.

Crusoe rechaza visceralmente el consumo de carne humana, así como aceptar las sobras de comida, ni siquiera con los alimentos rescatados. Esta reacción también demuestra que, a pesar de haber sido privado de alimentos civilizados y estar restringido a consumir frutos y animales en la isla-inicialmente en su estado barbárico-, estos no degeneraron su impulso alimentario. Su actitud hacia los restos del festín demuestra la predominancia de la civilidad que construyó por medio del cultivo, la ganadería y las técnicas de preservación y cocción, ya que al tener los cuerpos frente a él, se niega siquiera a probarlos. Dicha negativa no se presenta frente a los demás alimentos en la isla.

Crusoe suele realizar un proceso de cata de alimentos. Desde antes de llegar a la isla, muestra precaución antes de consumir el tigre que se encuentra junto con Xury; ("This ["lyon"] was Game indeed to us, but this was no Food", 22) así como varias aves cuando ya está en la isla ("I saw Abundance of Fowls, but knew not their Kinds, neither when I kill'd them could I tell what was fit for Food, and what not”, 40), pero no rechaza su ingesta por tratarse de productos barbáricos, sino porque no son aptos para el consumo. En el segundo caso, sin embargo, Crusoe se atreve a probar la carne del ave y "its Flesh was Carrion and fit for nothing" (40), lo que indica que consume los alimentos antes de etiquetarlos de inadecuados. Pero este proceso no es llevado a cabo con los restos del festín caníbal. En la novela, el canibalismo es la expresión alimentaria máxima del barbarismo porque implica asumir al ser humano como alimento y, a la vez, como devorador. Cuando Crusoe se niega a probar carne humana y califica a los participantes como salvajes, se cancela una actitud que hasta ese momento había sido constante: solo consumir alimentos después de probarlos y aceptarlos como tal. Al hacerlo, Crusoe sobrepone su civilidad y se ubica plenamente en el extremo civilizado de la dicotomía, en la que hasta ese momento se movía. 


\section{I2. Conclusiones}

La dieta de Crusoe presenta transformaciones que oscilan entre el barbarismo y la civilidad. Pero el enfrentamiento contra el canibalismo, tanto en consumo como en actitud, es lo que remarca su civilidad alimentaria. Esta se construye gradualmente y culmina cuando rechaza el canibalismo, que es en sí mismo la cumbre de la barbaridad. El proceso de civilidad se presenta cuando consume comida europea, cultiva granos, aprende a hacer pan, domestica cabras y sofistica sus platillos. Al domesticar sus alimentos también refleja la evolución de la humanidad al retratar procesos agricultores, ganaderos y culinarios en la isla. Los momentos de alimentación previos a su encuentro con los restos del festín caníbal alcanzan su punto máximo civilizado cuando Crusoe los rechaza como alimento.

Por otro lado, estos procesos intrínsecamente ligados a su consumo de alimentos devienen de una profunda devoción cristiana y el temor a los caníbales. Crusoe, al ser la imagen del protestante devoto, desprecia el catolicismo y las ceremonias que simulan el consumo del cuerpo de Cristo. La negativa a comer el cuerpo humano alegóricamente se ve reflejado en lo concreto en su repudio hacia los caníbales. Crusoe remedia su temor a lo salvaje por medio de la domesticación de animales y cocción de frutos silvestres, separando su dieta de aquella cuyo punto máximo es el canibalismo. Asimismo, Crusoe rompe con la costumbre de catar y aprobar alimentos al ver el festín caníbal, lo que demuestra que ha superado la necesidad alimentaria y puede discriminar las comidas barbáricas, a diferencia de lo que ocurría al inicio de su estancia.

A lo largo de este artículo y a través de la perspectiva alimentaria, hemos matizado la noción eurocéntrica de civilidad intrínseca en el personaje de Crusoe. Su alimentación ha requerido de un proceso de civilización o domesticación de los productos y de las técnicas para consumirlos. Es por ello que no se puede asumir que Crusoe, alimentariamente, sea la imagen de una civilidad innata. Estos procesos también se observan en la robinsonada, género literario cuyo nombre fue acuñado por Johann Gottfried Schnabel 
en 1731, que continúa hasta hoy y que está compuesta por obras inspiradas y derivadas de Robinson Crusoe. Estas reproducen una estructura similar a la del libro de Defoe: el protagonista aparece en un páramo desolado, debe rescatar comida, sembrar la tierra y cocinar alimentos.

Este artículo busca remediar el limitado alcance de los estudios alimentarios en obras dieciochescas cuya relevancia sigue vigente. La alimentación como objeto de estudio responde primera y elementalmente al realismo, ya que en la obra literaria, el alimentar a un personaje lo acerca a la existencia humana tal y como los lectores la entendemos. Por otra parte, los estudios alimentarios reflejan una continuidad tanto dentro de la obra de Defoe (ya que la comida es el único elemento presente durante toda la novela) y con las demás obras de la robinsonada, como, por ejemplo, las novelas The Road (Cormac McCarthy), The Martian (Andy Weir), la serie televisiva Lost (2004) y la película Cast Away (2000).

Estos ejemplos presentan la comida de formas puntuales, ya sea como festividad, como sucede con las papas en The Martian, compañía (Wilson en Cast Away) o recordatorio de la vida previa (las latas de comida en The Road). Así, a pesar de que las obras se transforman en contexto y en circunstancias (en cuestiones de la esclavitud, que ya no se presenta en la mayoría de las obras contemporáneas o el aislamiento que no está en Lost ni en The Road), todas comparten la necesidad alimentaria y su preponderancia en el desarrollo de la historia. En vista de que la obra de Defoe y la subsecuente robinsonada que han sido relevantes hasta nuestros días, aquí se propone una perspectiva de análisis todavía no explorada sobre una obra cuya importancia no ha disminuido desde su concepción. 


\section{Bibliografía}

Albala, Ken. Food in Early Modern Europe (Food through History). Santa Bárbara: Greenwood Press, 2003.

Avramescu, Cătălin. An Intellectual History of Cannibalism. Princeton: Princeton UP, 2003.

Bertrand, Didier. "Order and Chaos in Paradise: Colonial and 'Postcolonial' Constructions of Religious Identity through the Robinson Crusoe Story". Religion \& Literature, n. ${ }^{\circ} 3$, 1995, pp. 29-51.

Cole, Lucinda. Imperfect Creatures: Vermin, Literature, and the Sciences of Life, 16001740. Ann Arbor: Michigan UP, 2016.

Defoe, Daniel. Robinson Crusoe. Nueva York: W. W. Norton \& Company, 1994.

Durbach, Nadja. "Roast Beef, the New Poor Law, and the British Nation, 183463", Journal of British Studies, n. ${ }^{\circ}$ 4, 2013, pp. 963-89.

Fitzpatrick, Joan, editor. Renaissance Food from Rabelais to Shakespeare (Culinary Readings and Culinary Histories). Farnham: Ashgate, 2010.

Flynn, Christopher. "Nationalism, Commerce, and Imperial Anxiety in Defoe's Later Works", Rocky Mountain Review of Language and Literature, n. ${ }^{\circ} 2$, 2000, pp. 11-24.

Grumett, David. "Food and theology", Routledge International Handbook of Food Studies. Abingdon on Thames: Routledge, 2013. pp. 159-68.

"Husbandry". The Complete Farmer: or a General Dictionary of Husbandry. Londres: S. Crowder, 1766, p. 332.

LeBlanc, Ronald D. "Feeding a Poor Dog a Bone: The Quest for Nourishment in Bulgakov's Sobach'e Serdtse". The Russian Review, n. ${ }^{0} 1,1993$, pp. 58-78.

Lévi-Strauss, Claude. Structural Anthropology. Nueva York: Basic Books, 1963.

-. $\quad$ The Raw and the Cooked (Introduction to a Science of Mythology: 1). Nueva York: Pimlico, 1994.

McLean, Alice. "The Intersection of gender and food studies". Routledge International Handbook of Food Studies. Abingdon on Thames: Routledge, 2013, pp. 25064.

Montanari, Massimo. Food is Culture. Nueva York: Columbia UP, 2004. 
Newman, L. F. "Some Notes on Foods and Dietetics in the Sixteenth and Seventeenth Centuries". The Journal of the Royal Anthropological Institute of Great Britain and Ireland, n. ${ }^{\circ}$ 1, 1946, pp. 39-49.

Wheeler, Roxann. “'My Savage,' 'My Man': Racial Multiplicity in Robinson Crusoe”. ELH, n. ${ }^{\circ} 4,1995$, pp. 821-61. 